\title{
Modelling the performance of computer mirroring with difference queues
}

\author{
Przemyslaw Pochec \\ Faculty of Computer Science \\ University of New Brunswick, Fredericton, Canada E3A 5A3 \\ emailpochec@unb.ca
}

\begin{abstract}
The paper describes modelling of the performance of a special case of two computer systems operating in parallel: computer mirroring. In computer mirroring one of the systems is identified as the main and the other as the backup computer; both computers execute the same tasks. Each system operates independently and consists of an execution unit and a queue of tasks. Task scheduling and execution are modelled as Poisson processes. The performance of the mirroring system is modelled as a difference queue. The conditions for and the steady state solution for the performance of the computer mirroring system are derived. The processing rate $\mu_{2}$ of the backup system in computer mirroring should be no less than the product of the processing rate $\mu_{1}$ of the main system and the utilization rate $\rho_{1}$ of the main system, i.e. $\rho_{1} \mu_{1}<\mu_{2}$.
\end{abstract}

\section{INTRODUCTION}

Consequences of a failure of a computer system are not limited to just the cost of the necessary repairs, but include the cost of lost performance when the system is not operational. For mission critical applications where potential loss is exceptionally high, preventive measures aimed at increasing the overall reliability of the computing system can be undertaken. One such protection is the use of a second computer installation for backup. The backup computer performs the same operations as the main system, preferably at the same time. In case of the critical failure of the main system the required functionality is maintained by the backup system. This scenario is often referred to as computer mirroring since the backup computer "mirrors" the operation of the main system.

0-7803-4314-X/98/\$10.00 @1998 IEEE
It is most desirable for the backup computer to keep up with the processing speed of the main system. In practice uneven delays in the operation of both systems can be encountered resulting in the delays in completion of operations on the backup computer.

In this paper we analyze computer mirroring system from the point of view of delays in the operation of the backup system. A queueing network model for the operation of the mirroring system is developed, and estimators for the delays are derived.

\section{QUEUEING MODEL OF MIRRORING SYSTEM}

There is more to the speed of a computer system than just the processor clock rate. Instructions can execute only as fast as they can be read from memory, and this is different depending if they are already in the cache or still in the main storage. Access to data on disk depends again on caching and on where the data is physically stored on disk. On a multitasking system operations of one task can be preempted by another higher priority operation delaying the completion of the original task. It is clear that even for identical sets of instructions executing on identical hardware platforms we may expect some variation in execution times. For this reason we adopt a statistical model for analysis of the performance of a complex computer system.

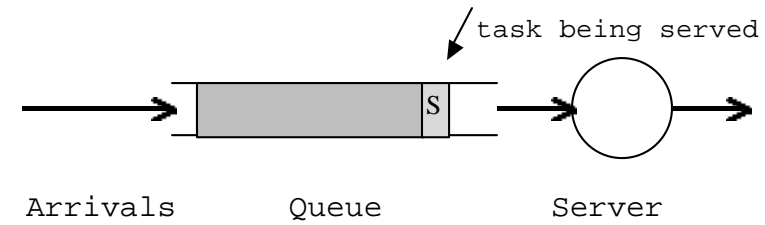

Figure 1. Queueing model of a computer system.

Operation of a computer system is modelled as a single server queue having random arrivals and random service. By random arrivals (and service) we assume that the probabilities of arrival over two infinitely small non overlapping intervals of length $\Delta t$ are the same. Such an arrival (and service) pattern 
is recognized as a Poisson process. Tasks scheduled for execution are placed (arrive) in the queue. Tasks are executed (served) in order of arrivals.
This queueing model is the well known model of the single server Poisson queue M/M/1. It is defined

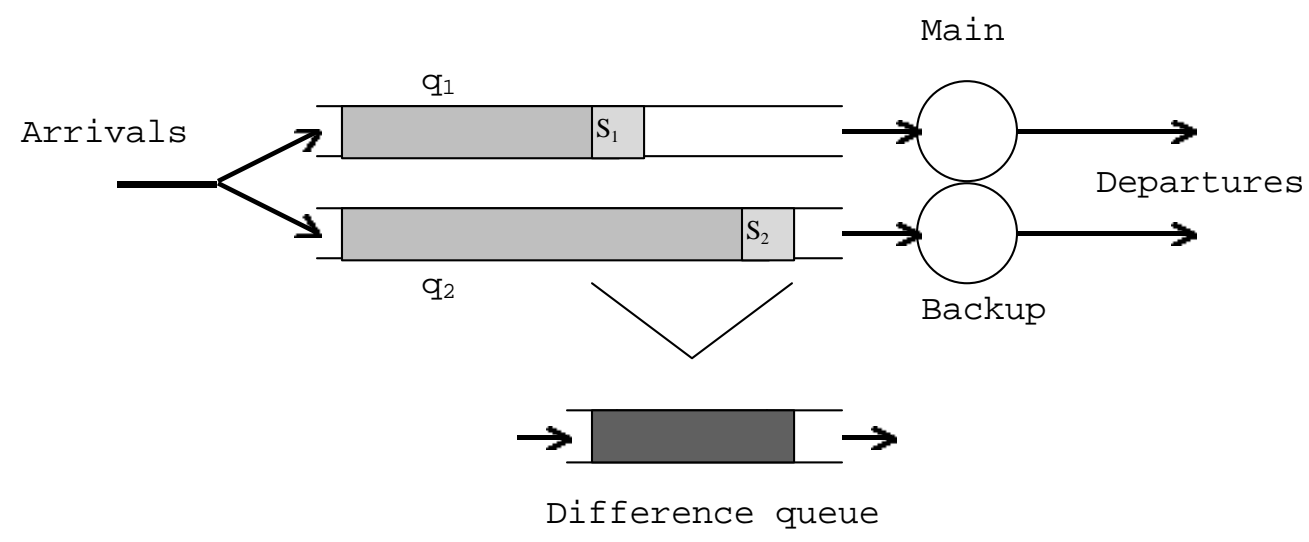

Figure 2. Queueing model of computer mirroring system.

with two parameters: the average arrival rate $\lambda$ and the average service (processing) rate $\mu$. The probability density functions for the interarrival and service times are exponential. The utilization of the system is defined as $\rho=\lambda / \mu$. Of particular interest for the discussion that follows is the probability distribution of the waiting time (including service) for the $M / M / 1$ queue:

$$
f(t)=(\mu-\lambda) e^{-(\mu-\lambda) t} .
$$

The mirroring system consists of two computer system units, each comprised of a queue and an execution unit. The tasks are scheduled (arrive) for both systems simultaneously and the arrival rate is modelled after the Poisson process. Execution of tasks on each of the computers is independent from the other. The average arrival rate is $\lambda=\lambda_{1}=\lambda_{2}$ and the service rates are $\mu_{1}$ and $\mu_{2}$ respectively. This system is very similar to the parallel network of queues described by Saaty [2] except that the same Poisson process generates arrivals to both queues. When referring to the lengths $L_{1}, L_{2}$ of queues $q_{1}$ and $q_{2}$ we shall account for the tasks being served, i.e. we will use the total number of items in the queueing system.
The statistical equilibrium equations for the steady state behaviour of the model of the mirroring system are difficult to solve in closed form. However the solution can be derived easily using the approach of difference queues.

\section{DIFFERENCE QUEUES}

The concept of a difference queue naturally follows from the queueing model of the mirroring system. During the parallel operation of two computer systems it is expected that the execution times for the individual tasks, and therefore queue lengths on each of the systems will differ slightly. Since in our analysis of the performance of the mirroring system we are interested in the delay of completion of a task on the backup system, we would like to estimate how much time is needed for the backup computer to catch up with the main system in case it has fallen behind. The backup system needs to execute the tasks that remain in its queue, and which have already departed (have been processed) from the queue in the main computer. Thus the concept of the difference between two queues arises.

It is interesting to note that the difference between the two queues defined above is also a queue. This queue, because of its origin, will be called the difference queue, or the $d$-queue. The arrivals to the 
d-queue are the departures from the queue $q_{1}$ on the main computer. (As the main computer finishes another task, the backup system falls behind by another notch, i.e. an element is added to the dqueue.) The departures from the d-queue are simply the departures from the queue $q_{2}$ on the backup computer. (Another task finishing execution on the backup computer reduces the disparity between the two queues.) The queueing discipline for the d-queue remains first come first serve.

The d-queues for modelling the mirroring system arising from two queues $q_{1}$ and $q_{2}$ with lengths $L_{1}$ and $L_{2}$ respectively can be classified into four types.

\begin{tabular}{|c|l|}
\hline Difference queues & \multicolumn{1}{c|}{ Conditions } \\
\hline Type 1 & $L_{1}, L_{2}=\infty, L_{2}-L_{1} \geq 0$ \\
\hline Type 2 & $L_{1}, L_{2}=\infty$ \\
\hline Type 3 & $L_{1}, L_{2}<\infty, L_{2}-L_{1} \geq 0$ \\
\hline Type 4 & $L_{1}, L_{2}<\infty$ \\
\hline
\end{tabular}

Types 1 and 2 correspond to cases when both system are always busy $\left(\rho_{1}, \rho_{2} \geq 1\right)$, i.e. queues $q_{1}$ and $q_{2}$ are never empty. Types 3 and 4 cover the cases when the queues may become completely depleted.

More important is the distinction between types 1, 3 and 2, 4. For defining types 1 and 3 we assume that the backup system can not overtake the main system, i.e. the length $L_{2}$ of the queue in the backup system can not be less than the length $L_{1}$ of the queue in the main system. This implies that the length of the $\mathrm{d}$-queue is non-negative. We will show that with nonnegative length, Poisson arrivals and departures the dqueues of type 1 and 3 are standard Poisson queues $\mathrm{M} / \mathrm{M} / 1$.

For types 2 and 4, with no restriction on relative lengths of queues $q_{1}$ and $q_{2}$, we have the most general case of d-queues allowing for negative queue lengths.

\section{DELAY JITTER IN COMPUTER MIRRORING}

In our analysis of computer mirroring we are going to place an additional restriction on the operation of the backup system: we disallow the backup system to complete execution of a task before the main system has finished executing it. For example, if we assume that the tasks arriving for execution are numbered sequentially $1,2, \ldots, n$, then the execution of task $i$ on the backup system can only start if the task $i-1$ has finished executing on the main system; if needed the backup processing unit will keep executing (idling) task $i$ intil the main unit finishes it. As a consequence the backup system can never overtake the main system, and the d-queue length remains non-negative. This in turn permits the modelling of the behaviour of the mirroring system with d-queues of types 1 and 3 . (Note that the queueing system of the backup computer is no longer a simple $\mathrm{M} / \mathrm{M} / 1$ queue.)

We define the delay in the computer mirroring system as the time from the instant the execution of a task completes on the main computer to the instant at which the execution of the same task terminates on the backup system. This definition is analogues to the definition of the waiting time (including service) for the d-queue discussed in the previous section.

\subsection{High Load Condition}

High load conditions occur when the main system remains busy at all times, i.e. its queue is never completely depleted of tasks scheduled for execution. This is typically the case for high utilization rates $\rho$, for example, when the main system is overwhelmed with high rate of arrivals compared with its processing capabilities. (The only desirable effect of such a condition is the minimization of the economic loss due to the main system idling.)

The delay of the backup system is derived from the behaviour of the d-queue of type 1 . The arrivals to the d-queue are the departures from the $q_{1}$ on the main system. They follow Poisson distribution and, since the $q_{1}$ is never empty, the arrival rate for the $\mathrm{d}$ queue is the same as the service rate $\mu_{1}$ of the main system. The departures from the d-queue are the Poisson departures from $q_{2}$ on the backup system with average service rate of $\mu_{2}$. The delay of the backup system is the same as the waiting time in the d-queue and has the probability distribution

$$
f\left(t_{H}\right)=\left(\mu_{2}-\mu_{1}\right) e^{-\left(\mu_{2}-\mu_{1}\right) t_{H}},
$$

and the mean

$$
E\left[t_{H}\right]=\frac{1}{\mu_{2}-\mu_{1}} .
$$

\subsection{General Case}

Under low load conditions $\left(\rho_{1}<1\right)$ the queue on the main system may become periodically empty, thus allowing for the backup system to catch up with the main system. It is expected to have a positive effect on reducing the delay of the backup system. 
For modelling such a scenario we will use a dqueue type 3 . The arrival for the d-queue is still the same as the departure from the queue $q_{1}$ on the main system, however the arrival rate is no longer defined as the processing rate $\mu_{1}$ of the main system. A new arrival to the d-queue occurs only when the queue on the main system is not empty. Recall that the probability of the $\mathrm{M} / \mathrm{M} / 1$ queue to be not empty is $P(N O T$ empty $)=\rho$. Consequently the probability of the new arrival to the d-queue over the interval $(t, t+\delta t)$, to the first order, is $\rho_{1} \mu_{1} \delta t$. The departures from the $\mathrm{d}$-queue are the Poisson departures from the $q_{2}$ on the backup system with average service rate of $\mu_{2}$. The probability of a departure is $\mu_{2} \delta t$. The stationary distribution can be derived from the following equilibrium equations

$$
\begin{aligned}
& \rho_{1} \mu_{1} P_{0}=\mu_{2} P_{1} \\
& \left(\rho_{1} \mu_{1}+\mu_{2}\right) P_{1}=\rho_{1} \mu_{1} P_{0}+\mu_{2} P_{2} \\
& \ldots \\
& \left(\rho_{1} \mu_{1}+\mu_{2}\right) P_{n}=\rho_{1} \mu_{1} P_{n-1}+\mu_{2} P_{n+1}
\end{aligned}
$$

where $P_{i}$ is the probability of having $i$ elements in the type 3 d-queue. Substituting $\rho_{1} \mu_{1}=\mu$ we get a set of equilibrium equations defining the $M / M / 1$ Poisson queue [1, page 40] with the arrival rate $\mu=\rho_{1} \mu_{1}$ and service rate $\mu_{2}$. Hence the probability density function for the delay in the mirroring system (i.e. waiting time in the type $3 \mathrm{~d}$-queue) is

$$
f\left(t_{L}\right)=\left(\mu_{2}-\rho_{1} \mu_{1}\right) e^{-\left(\mu_{2}-\rho_{1} \mu_{1}\right) t_{L}},
$$

and the mean is

$$
E\left[t_{L}\right]=\frac{1}{\mu_{2}-\rho_{1} \mu_{1}}, \quad \rho_{1}<1 .
$$

Note: Equations 5, 6 become equations 2, 3 for $\rho_{1}=1$.

\section{DISCUSSION}

A typical mirroring system with identical main and backup computers may experience significant delays in the operation of the backup system under moderate to high load conditions. When $\mu_{1}=\mu_{2}$ and $\rho_{1}$ is close to 1 the average delay defined in Equation 6 is high. For $\rho_{1}=1$ we have no steady state solution, and consequently no limits on the delays for the system defined in our model.

The performance of the mirroring system can be improved by increasing the processing rate of the backup system and decreasing the utilization $\rho_{1}$ of the main system so that

$$
\rho_{1} \mu_{1}<\mu_{2} .
$$

In fact since $\rho_{1}=\lambda_{1} / \mu_{1}$ and $\mu_{2}-\rho_{1} \mu_{1}=\mu_{2}-\lambda_{1}$ in Equations 5 and 6 the distribution of delays in the mirroring system under low load conditions $\left(\rho_{1}<1\right)$ depends only on the arrival rate $\lambda=\lambda_{1}=\lambda_{2}$ and the processing rate $\mu_{2}$ of the backup system. (Note that the backup system need not to be faster than the main system.) Changing the processing rate $\mu_{1}$ of the main system has no effect on the delay jitter in the mirroring system (steady state) under low load.

The relative speeds of the main and backup systems influence the delay in computer mirroring only under the heavy load operation $\left(\rho_{1} \geq 1\right)$. When the main system is continuously busy the delays are inversely proportional to the difference in processing rates $\mu_{2}-\mu_{1}$.

The $k^{\text {th }}$ percentile for the delay time $T_{0}$ derived from the density function in Equation 5 is

$$
T_{0}=\frac{\ln \frac{100}{100-k}}{\mu_{2}-\rho_{1} \mu_{1}} .
$$

For example: a computer mirroring system consisting of two computers with identical processing rates $\mu_{1}=\mu_{2}=100$ transactions per second, and the utilization of the main computer of $70 \%, \rho_{1}=0.7$, would experience delay of less than 0.154 seconds $99 \%$ of the time. If the utilization of the main system was increased to $\rho_{1}=0.9$, the delay of less than 4.6 seconds should be expected $99 \%$ of the time. Note that $1 \%$ of the time the delay may exceed the average processing time 460 times.

\section{CONCLUSION}

Performance of the computer mirroring system is directly linked to the behaviour of the d-queue, the difference queue derived from the queues on the main and backup computers. The steady state solution for the model exists for $\rho_{1} \mu_{1}<\mu_{2}$ or $\lambda<\mu_{2}$. Minimum delays in the operation of the backup system are obtained for $\lambda \ll<\mu_{2}$ under normal operating conditions (low load), and $\mu_{1}<<\mu_{2}$ for operation under high load.

\section{REFERENCES}


1. Cox, D. R., Smith, W. L., Queues, Methuen \& Co Ltd, 1967.

2. Saaty, T. L., Elements of Queueing Theory, McGraw-Hill, 1961.
3. White, J. A., Schmidt, J.W., Bennett, G.K., Analysis of Queueing Systems, Academic Press, 1975. 